\title{
Versión en chino de La casa de Bernarda Alba: la estrategia de fidelidad flexible y su valor como la primera traducción de teatro español al chino no simplificado
}

\author{
Menghsuan Ku \\ Universidad Nacional Chengchi \\ menghsuanku@hotmail.com
}

Recibido: 19 de noviembre de 2014

Aceptado: 27 de marzo de 2015

\section{RESUMEN}

El objetivo principal de este trabajo consiste en indagar en el concepto de traducción que muestra el traductor chino Perng Ching-hsi ${ }^{1}$ (1945- ). Para ello, comparamos las traducciones de la obra de teatro La casa de Bernarda Alba en inglés, en chino y el texto original en español, con las reflexiones sobre la traducción que propone el propio traductor. Como objetivo específico nos planteamos justificar la flexibilidad dentro de la fidelidad a una traducción. Así pues, por un lado, hemos detectado elementos locales de la cultura española que nos despiertan el interés a la hora de comprobar su resolución; así como investigar las técnicas y estrategias aplicadas por el traductor (tanto referentes al aspecto de nombres propios de personas como al de expresiones). Por otro lado, enumeramos a continuación los errores de traducción relativos al conocimiento cultural y la lengua detectados en las traducciones.

Palabras clave: elemento cultural, error de traducción, García Lorca, chino, taiwanés.

The Chinese Translation of La casa de Bernarda Alba: The Strategy of Flexible Faithfulness and the Significance of the First Spanish Play Translated into Traditional Chinese
ABSTRACT
This work aims to discuss the concept of translation proposed by the Chinese translator Perng Ching-hsi. In order to achieve that goal we compare the English and Chinese transla-

\footnotetext{
1 Perng es actualmente Profesor Emérito de la Universidad Nacional de Taiwán (NTU). Más información Ref. 4.2. Nociones sobre las traducciones. 
tions of the Spanish theatre play The House of Bernarda Alba. The specific purpose is to justify the flexibility of faithfulness in Perng's translation. Therefore, on the one hand, with regard to the interesting Spanish cultural elements, this work analyses resolutions and investigates translation skills and strategies employed: terminology of people's names as well as expressions. On the other hand, we point out translation mistakes caused by cultural and language differences.

Keywords: cultural element, translation mistake, García Lorca, Chinese, Taiwanese.

Sumario: 1. Introducción. 2. Lenguaje del escenario. 3. Problemas y errores de traducción. 4. La casa de Bernarda Alba en chino. 4.1. Traducciones de La casa de Bernarda Alba. 4.2. Nociones sobre las traducciones. 4.3. Soluciones de traducción. 5. Conclusiones.

\section{Introducción}

La traducción de La casa de Bernarda Alba constituye una clave ineludible a la hora de abordar la evolución de la traducción de la literatura española al chino. Esta traducción al chino es pionera dentro de la literatura española; apareció formando parte de una serie de traducciones de teatro occidental y contiene los datos completos del texto intermedio inglés de donde se tradujo. Aunque La casa de Bernarda Alba es una traducción indirecta, el traductor Perng Ching-hsi es un traductor de larga experiencia que ha redactado unos artículos con sus reflexiones en cuanto a la tarea traductora. Todos estos factores nos estimulan a realizar una investigación objetiva para presentar la situación de la traducción de la literatura española al chino en su época más temprana.

Hay pocos comentarios o reflexiones sobre la traducción al chino de La casa de Bernarda Alba. Sin embargo, en la introducción de las traducciones de Der gute Mensch von Sezuan y Der kaukasische Kreidekreis publicadas en chino en 2005, Perng menciona en dicha introducción su preocupación sobre las carencias inevitables de una traducción indirecta (Brecht 2005: 1), que es el caso de todas sus traducciones de autores de habla no inglesa. Para las traducciones de Bertolt Brecht que hizo Fang-hsiung Dscheng, de dos obras de teatro, el traductor usó la traducción de Der gute Mensch von Sezuan de Perng ${ }^{2}$ como borrador. Debido a que la versión de Perng proviene de la traducción inglesa de Eric Bentley, además de una corrección necesaria, Dscheng ha añadido bastantes partes omitidas (Brecht 2005: 6-7), aunque Dscheng también ha alabado la lealtad de la traducción de Perng y el buen nivel de chino que tiene (Brecht 2005: 7). Por tanto, las traducciones indirectas constituyen un campo de estudio específico, debido a que en el proceso traductor intervienen factores de doble complejidad.

${ }^{2}$ Brecht, Bertolt. 布雷希特戲劇選集, 四川好人 [Antología de las obras de Bertolt Brecht, La buena mujer de Sichuan], trad. por Perng Ching-hsi en Yan Yuan-shu 顏元叔. En: 淡江西洋現代戲劇譯丵 Serie de traducciones de los teatros occidentales modernos del Colegio Tamkang, Taipei: Jingsheng 驚聲 1973. 


\section{Lenguaje del escenario}

A diferencia de otros géneros literarios, el teatro es una combinación de lengua y representación. Debido a esta característica integral, no es de extrañar que a finales de los años setenta, Bassnett comentara que un texto de teatro por sí solo está incompleto (Bassnett 1978: 161-176) a consecuencia de los varios elementos que forman una obra (la luz, el escenario, la música, etc.) además del guión. De ahí que la traducción del teatro se considere más complicada que cualquier otro texto en el sentido de que traspasa el formato puramente escrito. Por tanto, Lefevere (1980: 158) comentó la escasez de teorías de traducción del drama, aunque, poco a poco se han ido incorporando más estudios a lo largo de las últimas décadas, lo que ha permitido avanzar en el desarrollo de otras ramas de la traductología.

A pesar de que el teatro se caracteriza por una presentación integral, el aspecto escrito de un guión ya es por sí mismo un texto particular. Para un análisis más concreto enfocamos nuestro trabajo al estudio de la traducción del guión sin entrar en el arte de la representación teatral. Observando los trabajos preparatorios de la traducción de un guión, consideramos que el trasfondo sociocultural, los dialectos y los elementos propios de una cultura, son factores sumamente importantes que deben tratarse con suma precaución en la tarea traductora. Puesto que estos elementos culturales son mecanismos para reflejar las ideas del dramaturgo y el trasfondo de la historia, ofreciendo, además, el espacio y el tiempo para una imaginación concreta a los lectores y espectadores. En segundo lugar, dado que el texto íntegro del guión está en forma de diálogo, la cohesión juega indudablemente un papel importante a fin de crear conversaciones lo más fluidas posible. A nuestro parecer, estos dos aspectos son los esenciales que el traductor debe tener en cuenta al revisar el producto traslativo.

La importancia del papel de la cultura en la traducción teatral lo podemos observar en un ejemplo que menciona Anderman (1998 y 2001: 72). El autor indica que en el trabajo de Ooi (1980) este comentaba sobre la cuestión cultural en una obra de O'Neill representada en China, donde los papeles familiares son claros y no permiten ningún desafío. De hecho, como "its subtextual ambiguity and multiple tonality are still new to us in Hong Kong" (Ooi 1980: 60), tanto la ambigüedad de la relación familiar como la de ironía resultan un reto para el traductor y la audiencia china. Mientras tanto, en Aaltonen (2000) la autora ha propuesto tres perspectivas de estudios sobre la traducción teatral, a saber: el lingüístico, el teatral, el socio-histórico y cultural. La autora en su tesis también ha incidido en el campo cultural, ha introducido la noción de manipulación y ha recalcado la prominencia de la cultura en la traducción del teatro.

Así, si observamos la tendencia de colocar el proceso traslativo de una lengua y cultura a la otra, es imprescindible tomar en consideración el marco del trasfondo de la sociedad y las costumbres. Es cierto que como texto multidimensional, el guion teatral tiene varios aspectos que se pueden estudiar. Por otro lado, los problemas acaecidos durante la traducción pasan tanto en dos culturas lejanas como en dos cercanas porque las dos culturas cercanas pueden poseer costumbres similares pero no coincidir totalmente, y viceversa. 
En nuestra opinión, la traducción de elementos culturales es un tema general en todo tipo de traducciones aunque en nuestro caso, la traducción teatral, resulta mucho más aparente debido a las escenas concretas y a las conversaciones cotidianas.

\section{Problemas y errores de traducción}

En este apartado tratamos del concepto de errores de traducción y diversas nociones al respecto. De hecho, la traducción de Perng era una traducción de segunda mano, es decir, no directamente del castellano sino a través de las traducciones del texto en inglés. En su caso, se ha basado en la traducción de James GrahamLujan y Richard L. O'Connell, publicada en 1941. Por eso, cabría la duda de si resulta una traducción sin faltas respecto a la versión original de Lorca, aunque nuestra intención no es encontrar detalladamente los errores cometidos durante este proceso traslativo indirecto sino aclarar las estrategias y las técnicas aplicadas por Perng al enfrentarse a los problemas de traducción.

Los errores o las faltas provienen de las dificultades y problemas que no se supieron solventar adecuadamente. Las dificultades son subjetivas, ya que cada uno se enfrenta con dificultades distintas, mientras que los problemas son objetivos y existen a pesar de las competencias traductoras de los traductores. (Nord 1988 y 2005: 151). Por eso, sea cual sea la clasificación de los problemas de traducción, siempre hay aspectos o cuestiones por las que los teóricos se preocupan por igual. Nord (1988 y 2005: 151) divide los problemas en cuatro categorías: los problemas textuales, pragmáticos, culturales y lingüísticos. Las propuestas de Hurtado (2001: 288) también son de cuatro tipos: lingüísticos, extralingüísticos, instrumentales y pragmáticos. Aunque siempre hay matices en cuanto al contenido de cada categoría entre las clasificaciones de las dos autoras, la coincidencia entre sí nos ha proporcionado bastante información sobre los aspectos de la lengua y de la cultura que conducen con mayor probabilidad a problemas en general.

En cuanto a la clasificación de los errores de traducción, podemos observar que al alejarse de una perspectiva estrechamente relacionada con la lengua, las reflexiones de las clasificaciones de errores son diversas y funcionales, tales como el binarismo de Pym (1992), que divide los errores entre binarios y no binarios. Los últimos ofrecen más posibilidades en una traducción aparte de lo correcto o no correcto (estos serían los binarios). Mientras la adopción del término "adecuación" de Kussmaul (1995) en los aspectos cultural, situacional, de los actos de habla y del significado de las palabras también revelan una actitud madura y tolerante a la hora de valorar la traducción. Correspondiéndose con la clasificación de los problemas de traducción, en la clasificación de Nord (1996: 98) hemos visto que hay tres tipos de errores: errores pragmáticos, errores culturales y errores lingüísticos. A nuestro parecer, la clasificación de Nord (1996) presenta otra dimensión objetiva y práctica en la evaluación de las traducciones, lo que resulta relativamente aplicable en el plano práctico. 


\section{La casa de Bernarda Alba en chino}

\subsection{Traducciones de La casa de Bernarda Alba}

En el año 1969, la Escuela Universitaria de Ciencias y Letras Tamkang fundó el Laboratorio de Literatura Occidental, cuyos objetivos son, por un lado, publicar en inglés la revista de estudios de literatura Tamkang Review y por el otro lado, traducir obras occidentales famosas (Yan 1973: 1). La publicación de la serie de The Tamkang Collection of Modern Western Plays in Chinese Translation es el fruto de su plan de traducción. Como primera tarea de traducción literaria los miembros del Laboratorio llegaron a un consenso para presentar obras teatrales importantes al pueblo de habla china. Después de un largo tiempo para confeccionar un listado de obras teatrales según su plan introductorio, al final quedaron más de cuarenta dramaturgos y sus obras más representativas ${ }^{3}$.

Las dos obras de Lorca seleccionadas en esta serie de traducción son Bodas de Sangre. Tragedia en tres actos y siete cuadros y La casa de Bernarda Alba. Drama de mujeres de los pueblos de España, traducidos por 王秋桂 [Wang Chiu-Kuei] y 彭鏡禧 [Perng Ching-Hsi] respectivamente. Son las dos primeras obras de Lorca traducidas al chino no simplificado. Debido a que el traductor está especializado en literatura inglesa ha partido de la versión inglesa de James Graham-Lujan y Richard L. O'Connell, The House of Bernarda Alba. A Drama about Women in the Villages of Spain para realizar la traducción al chino que ha quedado con el título resumido: 白納德之屋 [La casa de Bernarda]. Hasta hoy en día, esta versión indirecta es todavía la única traducción al chino publicada en Taiwán. Aunque más tarde, el Instituto de Posgrado de la Universidad Fujen publicó varias traducciones de literatura española traducidas directamente del español, y también hay traducción de la otra obra de Lorca, Yerma; pero no hay traducción directa de Bodas de Sangre ni de La casa de Bernarda Alba.

\subsection{Nociones sobre las traducciones}

Perng es doctor en Literatura Comparada por la Universidad de Michigan, realizó investigación posdoctoral en la Universidad de Yale, ejerció de profesor visitante en la Universidad de Virginia, de investigador visitante en la Universidad de

\footnotetext{
3 Según nuestra observación, el resultado no alcanza su plan inicial, puesto que los dramaturgos finalmente traducidos y publicados no llegan a cuarenta en total: Harold Pinter (1930-2008), Henrik Ibsen (18281906), Arthur Miller (1915-2005), John Millington Synge (1871-1909), Jean Paul Sastre (1905-1980), Edward Albee (1928-), Thomas Stearns Eliot (1888-1965), Ernst Ingmar Bergman (1918-2007), Eugene Gladstone O’Neill (1888-1953), Sean Ocasey (1884-1964), George Bernard Shaw (1856-1950), Samuel Beckett (1906-1989), Bertolt Brecht (1898-1956), Albert Camus (1913-1960), Thornton Niven Wilder (18971975), Jean Cocteau (1889-1063), Jean Anouilh (1910-1987), John Osborne (1929-1994), Edmond Rostand (1868-1918), Luigi Pirandello (1867-1937), Eugene Ionesco (1909-1994), Lorraine Hansberry (1930-1965), William Butler Yeats (1865-1939), Federico García Lorca (1898-1936), Tennessee Williams (1911-1983), Anton Pavlovich Chekhov (1860-1904).
} 
Oxford y en la Universidad de Chicago. De 1993 a 1996 fue director del Departamento de Lenguas Extranjeras en la Universidad Nacional de Taiwán (NTU) ${ }^{4}$ y de 2002 a 2005 fue Decano de la Facultad de Artes y Humanidades de NTU. Debido a su especialidad en el teatro de Shakespeare, le invitaron a hacerse cargo de la dirección del Departamento de Drama y Teatro de la NTU entre 2001 y 2003. Se retiró en 2009 y hoy día es Profesor Emérito del Departamento de Lenguas Extranjeras de la NTU. Aparte de las obras de Shakesperare, Perng ha mostrado un interés especial por la traducción literaria y tiene publicadas numerosas traducciones, la mayoría de teatro, aunque también poemas y narrativa ${ }^{5}$.

Siendo un traductor con bastante experiencia práctica, Perng constituye un claro ejemplo de la mentalidad general de depreciación de las traducciones. Con unos ejemplos entenderemos cómo incluso en el campo académico las traducciones no son valoradas suficientemente en Taiwán (Perng 1997: 7): los profesores universitarios no pueden subir de categoría a través de la publicación de traducciones; los alumnos de máster no pueden graduarse con traducciones en vez de un trabajo de investigación, a pesar de la alta calidad del texto original o del texto meta. El conflicto surge cuando en clases de literatura europea se utilizan las traducciones al inglés, pero el Departamento de Lenguas Extranjeras no ha considerado incluir en su itinerario curricular clases de literatura a través de las traducciones. Todo esto revela el escaso valor que se otorga a las traducciones en Taiwán. En cuanto al último aspecto que menciona Perng sobre utilizar traducciones inglesas con la finalidad de apreciar la literatura extranjera, no es una realidad que nos extrañe, porque hace veinte años no era posible encontrar muchas traducciones en inglés y menos aún en otras lenguas extranjeras consideradas minoritarias en Taiwán.

En las obras de Perng, el prólogo del traductor no se extiende demasiado sobre ningún aspecto de la traducción; no obstante, podemos observar las ideas que tiene sobre la traducción de teatro a través de sus reflexiones publicadas en Translating Hamlet, the Heart Way (2005) y Comentarios de traducciones literarias (1997). Listamos a continuación sus criterios de traducción:

a. La característica temporal y la necesidad de retraducir un texto. Como las traducciones se ven afectadas por el trasfondo social de la época, los textos originales requieren una renovación según diferentes contextos. Dicha necesidad de retraducir, de hecho, es una prueba del valor clásico y eterno del texto original. El hecho de retraducir en cada época con dife-

\footnotetext{
4 Ref. Página web del Departamento de Lenguas Extranjeras de NTU: http://www.forex.ntu.edu.tw/ main.php. Facultad de Artes Liberales de NTU: http://liberal.ntu.edu.tw/. Departamento de Drama y Teatro de NTU: http://homepage.ntu.edu.tw/ theatre/.

5 Las traducciones publicadas cronológicamente por Perng son: La casa de Bernarda Alba de García Lorca en Antología teatral de Lorca (1970); Endgame de Samuel Beckett en Antología teatral de Beckett (1970); Antología de cincuenta poemas cortos de Inglaterra y EEUU (1989), en colaboración con Yiangsheng Xia; Irrational Man de William Barrett (1969); Hamlet de Shakespeare (2001), Antología de narrativa taiwanesa (traducida del chino al inglés); Death in a corn field (1994), To a Daughter Leaving Home, and Other Poems, antología de poemas de Linda Pastan (2002); Der gute Mensch von Sezuen, Der kaukasische Kreidekreis de Bertolt Brecht (2005).
} 
rentes traductores asimismo favorece una mayor exactitud en la interpretación 6 .

b. La necesidad de mantener el estilo del texto original. Perng (1995: 33) menciona con un ejemplo de traducción en poesía que muchos poetas con talento traducen poemas que quedan mejor que las versiones originales. A nuestro parecer, debido a que cada traductor sigue su estilo personal, la modificación en mayor o menor grado es inevitable, sobre todo cuando el criterio del estilo es un elemento invisible. Ahora bien, otra cosa es que hoy en día los traductores se consideren con más poder sobre sus traducciones y puedan gestionar la operación textual como mejor consideren.

c. La insistencia en salvaguardar el contenido del texto original. Esta idea trata del contenido de una traducción como otro aspecto simétrico del último criterio, que trata del formato. Con su abundante experiencia en traducción de Shakespeare, Perng no olvida mencionar que los traductores deben traducir los mecanismos de juegos de palabras con la misma naturalidad que en el texto original. Por ejemplo, Hamlet contiene numerosos homófonos con muchas connotaciones, Perng ha intentado traducirlos al chino usando también los mecanismos fonéticos.

d. La misma fluidez en la traducción que en la obra teatral original. En cuanto a la traducción de una obra teatral, Perng insiste en que debe ser tan fluida como el texto original para ajustarse a la presentación en un escenario. Aunque confiesa que, de hecho, esto no es el único aspecto que requiere cualquier buena traducción; las obras de Shakespeare contienen otros factores a tener en cuenta en el momento de la traducción. Sin embargo, entendemos que una valoración o una crítica traductora abarca la relación estrecha entre la traducción y la manera de representarla.

e. La traducción de los nombres de los personajes favorece la representación teatral y la caracterización de los papeles. En cuanto a los nombres que aparecen en una obra, Perng (2005: 15) suele recortar los nombres en no más de tres caracteres, es decir, tres sílabas para convenir al efecto teatral. En caso de la aplicación de una estrategia de extranjerización, basta escoger caracteres poco usados en nombres chinos. ${ }^{7}$ En cuanto a los nombres con significado extra, Perng (2005: 15) ha tomado como ejemplos los nombres de personajes en obras de Shakespeare, para recordar la precaución que deben tener los traductores debido a la connotación que pueden tener estos nombres aparentemente normales: Angelo en Measure for Measure como un

6 Perng (2005: 32) señala que el significado de la palabra "fat" en Hamlet no se refiere a "sweaty" o "out of condition" como en la traducción de Jenkins (Shakespeare de la versión Arden) y en la mayoría de las otras versiones, sino quiere decir "very strong and able in combat", con el objetivo de revelar la necesidad de reinterpretar un clásico.

7 Ejemplos de nombres extranjerizados en traducción al chino son Rosencrantz traducido al chino como “羅增愷” [Luo Zenkai] y Guildenstern “紀思騰” [Ji Siteng] (2005: 15), aunque a nuestro parecer las dos traducciones no han conseguido totalmente la sensación de lejanía debido a su formato como nombres chinos de tres caracteres. 
ángel caído; Malvolio en Twelfth Night representando una persona de mal carácter y malas intenciones; Fortinbras en Hamlet que es un hombre fuerte de carácter. De esta forma, Perng prefiere traducir guardando tanto el formato como el contenido del texto original, es decir, salvaguardando su actitud de lealtad hacia el texto original. Por el otro, según las traducciones de los nombres propios de Perng y nuestra lectura del contenido de las obras traducidas, hemos percibido que sus traducciones tienen una tendencia a la localización hacia la cultura meta, es decir tienden a la lengua y a la cultura china.

\subsection{Soluciones de traducción}

En este apartado, enumeramos los fenómenos particulares en la traducción de $L a$ casa de Bernarda Alba en chino. Lo hemos dividido en cuatro secciones según el tema central de cada subapartado. En primer lugar, presentamos la traducción de los nombres de los personajes, que es un tema que todavía requiere más investigaciones empíricas. Sobre todo, hay muy pocos estudios que aborden la traducción de nombres entre el chino y las lenguas occidentales. Como en los artículos de Perng, este autor ha tratado la misma cuestión, consideramos necesario estudiarla con precaución. Luego introducimos el tema de la traducción de expresiones, que tienen cierto grado de dificultad debido a la distancia cultural. La claridad de la connotación de estas expresiones ya depende de la estrategia del traductor. Al final presentamos los errores detectados en la traducción de Perng. Y para una presentación clara, detallamos los fallos comprobados que atañen a la cultura y que mantienen una relación estrecha con la lengua.

\section{- Los nombres de personas revelan la tendencia de una traducción}

Los nombres de personas son unidades pequeñas en la tarea traductora y no resultan tan evidentes como los elementos de unidades mayores que afectan más a un texto ${ }^{8}$. Sin embargo, las traducciones pueden producir distinta sensación según la estrategia traductora aplicada a cualquier unidad. La traducción de los nombres propios entre el chino y las lenguas occidentales es un campo por estudiar. Este tema resulta más complicado que una traducción entre nombres occidentales básicamente debido al diferente sistema lingüístico del chino. En chino cada carácter es una sola sílaba, y una palabra puede estar formada por uno o dos caracteres, mientras que los nombres occidentales son de varias sílabas. Así, en el momento del proceso traslativo, uno puede escoger cualquier sílaba en chino y los caracteres que consi-

${ }^{8}$ Entre las propuestas de las unidades de traducción basadas en diferentes concepciones de la óptica lingüística, textual, cognitiva, etc. nuestra clasificación se centra en la perspectiva lingüística que diferencia palabra, sintagma y oración. 
dere más apropiados, ya sean correspondientes al carácter de la persona o a las reglas de eufonía. Luego hay más consideraciones como las costumbres convencionales del orden del apellido y el nombre ${ }^{9}$; pero este mecanismo ya es menos complicado que la traducción de las palabras.

En La casa de Bernarda Alba traducida por Perng, los nombres de personajes se plasman en chino con trascripciones fonéticas de nombres completos. Hemos confeccionado el cuadro para facilitar la comparación del nombre en castellano y su traducción al chino:

\begin{tabular}{|l|l||l|l|}
\hline Nombres en español & Traducciones en chino & Nombres en español & \multicolumn{1}{|c|}{ Traducciones en chino } \\
\hline Bernarda & 白納德 & Antonio María Benavides & 安東紐・馬里亞・白納維底斯 \\
\hline Marí Josefa & 瑪麗亞 ・何賽華 & Darajalí & 戴拉哈利 \\
\hline Angustias & 安珂斯蒂 & Paca la Roseta & 芭佳羅色德 \\
\hline Magdalena & 瑪德麗娜 & Maximiliano & 馬西米力亞諾 \\
\hline Amelia & 愛密麗亞 & Don Arturo & 唐阿都若 \\
\hline Martirio & 瑪蒂麗娥 & Adelaida & 艾黛萊德 \\
\hline Adela & 愛德拉 & Enrique Humanas & 昂里胡曼納 \\
\hline La Poncia & 潘霞 & Pepe el Romano & 斐貝羅曼諾 \\
\hline Prudencia & 卜丹霞 & Evaristo el Colín & 短尾依伐利斯多 \\
\hline Tronchapinos & 托嘉畢諾斯 & Librada & 李芭德 \\
\hline
\end{tabular}

Como el traductor ha hecho una traducción de los nombres completos a modo de transcripción, podemos ver que las sílabas corresponden exactamente con las de los nombres en español. En el momento de escoger los caracteres de estos nombres, el traductor los ha elegido de sonido femenino o masculino según el sexo de cada personaje. Esta consideración favorece la identificación de los personajes a lo largo de la lectura.

Sin embargo, en cuanto al formato de los nombres traducidos no hay una regla unánime para nombres de más de dos palabras. Las traducciones de "María Josefa" (瑪麗亞) y de “Antonio María Benavides” (安東紐・馬里亞・白納維底斯) en chino están separadas con puntos que corresponden con los espacios del texto original. Pero “Enrique Humanas” (昂里胡曼納) y “Pepe el Romano” (斐貝羅曼諾) no están separados, sino que los nombres y los apellidos van juntos. Como los nombres chinos cuentan con dos o tres sílabas, los nombres largos resultan excesivos. Aunque se encuentren dos nombres separados por puntos, para los lectores chinos es difícil de evitar la sensación de que algo sobra. ${ }^{10}$

Y si observamos las traducciones desde la perspectiva semántica, suenan bastante exóticos estos nombres traducidos excepto 潘霞 (La Poncia; pinyin: Panxia)

9 Cf. Moya, V. (2000), La traducción de los nombres propios, Madrid: Cátedra, p. 86, donde destaca un pequeño párrafo comentando la cuestión del orden del apellido y el nombre en chino a pesar de que hay otras consideraciones más importantes que podrían intervenir en las traducciones entre nombres españoles (occidentales) y chinos. Esto revela que es un campo aún por estudiar. A nuestro parecer, se trata de un tema interesante y merece más investigación tanto de aspectos empíricos como teóricos. 
y 李芭德 (Librada; pinyin: Li Bade) porque los dos apellidos, Pan y Li son comunes en chino. La protagonista, Bernarda, ha sido traducida como 白納德 . La primera letra, que sería como su apellido chino, coincide con la del nombre chino del protagonista masculino de la novela Lo que el viento se llevó, 白瑞德 (Rhett Butler). Esta coincidencia casual contribuye al efecto de una imagen extranjera. Este ejemplo de los dos nombres (Bernarda Alba vs. Rhett Butler) cuyos apellidos no tienen nada que ver pero que acaban con traducciones casi iguales en chino (白納德 vs. 白瑞德) refleja las posibilidades variadas en la traducción de nombres extranjeros al chino.

Estos fenómenos que hemos observado en la traducción de los nombres propios de personas no se corresponden con los criterios de Perng (2005) en el apartado 4.2., donde decía decantarse por la estrategia de recortar los nombres hasta tres sílabas o menos para facilitar la representación en el escenario. Mientras que la elección de los caracteres de La casa de Bernarda Alba no trata de reflejar los caracteres de los personajes sino que resultan meras trascripciones fonéticas acomodadas al sexo de cada personaje.

\section{- Expresiones que requieren una ampliación lingüística}

Debido a las diferentes convenciones de la cultura lingüística, las expresiones varían entre las lenguas. En La casa de Bernarda Alba, Lorca emplea expresiones populares andaluzas. Resulta poco factible que se entiendan bien si traducimos literalmente estas expresiones debido a las diferentes connotaciones de la cultura receptora.

Algún ejemplo se aclara por el contexto, porque en las conversaciones se explican las ideas aunque sean expresiones autóctonas occidentales. En la cultura española y la anglosajona existe la creencia de que si se derrama la sal habrá mala suerte: "Derramar el vino es buena señal, pero no la sal", dice el refranero. Sin embargo en China no existe la misma costumbre, aunque se tira un abanico o se echa agua hacia el coche de los novios para reflejar la separación entre la novia y su familia original ${ }^{11}$, pero no se arroja sal. Así pues, cuando Amelia dice a Magdalena, "Ya has derramado la sal [...] Siempre trae mala sombra." (Lorca 1954: 907) y en la traducción en inglés, "You've spilled the salt! [...] It always brings bad luck." (Lorca 1941 y 1955: 198) en la traducción china Perng opta por la traducción literal, “你把鹽溜掉了 [...] 它總是帶來壞運氣” (Lorca 1970: 184); sin la segunda oración, los lectores de la traducción en chino no podrían imaginar la relación entre las dos oraciones.

\footnotetext{
10 Asimismo, hemos notado una tendencia emergente en la traducción de nombres reflejada en las traducciones de novelas contemporáneas hispanoamericanas al chino. El protagonista de Tan veloz como el deseo de Laura Esquivel, Júbilo, es traducido al chino como “阿喜 (axi)”; “里八 (liba)”: Jesús González de la Riva de La familia de Pascual Duarte de Camilo José Cela, un nombre de cinco palabras se convierte en una palabra de dos sílabas en chino; Daniel de La sombra del viento, traducido como “達尼 (dání)” en chino omite la última sílaba, etc. El uso de nombres concisos es otra opción frente a la trascripción completa de todas las sílabas.

11 La costumbre china viene del sonido similar entre “separación” y “abanico”: “散 [sàn]” y “扇 [shàn]"; por otro lado está la imagen de que el agua tirada no se recupera se usa para trasmitir el deseo de un buen matrimonio y que la hija (novia) no vuelva a la casa de sus padres por problemas en su propia familia recién formada.
} 
En caso de que se trate de una expresión no contextualizada, como durante una pelea entre Adela y la criada Poncia, Adela replica, "No por encima de ti, que eres una criada; por encima de mi madre saltaría para apagarme este fuego [...]" (Lorca 1954: 879) y en la traducción en inglés, "For I'd leap not over you, just a servant, but over my mother to put out this fire [...]" (Lorca 1941 y 1955: 182- 183), una traducción literal en chino resulta poco fluida, debido a que en chino no se usa esta connotación. Por tanto, “我不只跳過你這個僕人, 我要跳過我母親 [...]” [No salto por encima de ti, que eres una criada; por encima de mi madre saltaría para apagar este fuego] (Lorca 1970: 160-161) sonaría extraño y quedaría mejor una traducción del sentido como “我會不計一切平息這慾火。不僅爲此殺了妳，你只是個僕人, , 我甚至連母親也不放過.”

Otros ejemplos de expresiones españolas aluden al tema de la religión. Hoy en día Taiwán es un país multirreligioso, en el que se sigue el budismo, taoísmo, cristianismo, e incluso el islamismo. No obstante, la mayoría de los taiwaneses profesa la religión popular de Taiwán, mientras que las otras religiones son importadas y cuentan con menos practicantes. Por tanto, es de imaginar que los ritos de las otras religiones no resulten familiares para los taiwaneses. Si se encuentran términos propios de aquellas religiones, es mejor traducirlos de manera más general sin afectar a la idea original o añadir información para que la entiendan los lectores chinos. Como por ejemplo "De pie y cantando al modo gregoriano" (Lorca 1954: 847), en inglés "standing and chanting in the Gregorian fashion" (Lorca 1941 y 1955: 163), en chino también se ha traducido literalmente “站起, 以唱哥利葛里聖歌的方式吟唱” [De pie y cantando al modo gregoriano como canción sagrada] (Lorca 1970: 133). Sin embargo, los lectores chinos ignoran qué es el "modo gregoriano", la ampliación de añadir "canción sagrada" o "canto medieval" facilitaría la comprensión lectora aunque la traducción fonética del "gregoriano" siga siendo una palabra incomprensible. Como el contexto ya se refiere al catolicismo, resultaría más comprensible si en la traducción al chino aplicamos la ampliación de la "canción sagrada medieval" que no existía en el texto original y eliminamos el término "gregoriano" que es poco familiar para los lectores chinos: 站起, 以唱中世紀聖歌的方式吟唱 [De pie y cantando al modo de canción sagrada medieval]).

Esta técnica de ampliación lingüística facilita la lectura y aumenta la comprensibilidad, por eso con frecuencia es recomendable usarla para traducir elementos propios de la cultura del texto original. Por ejemplo, "Cae el sol como plomo" (Lorca 1954: 844) se ha traducido al chino literalmente “太陽照下來像鉛一般” [Cae el sol como plomo] (Lorca 1970: 131). Sería más clara traducirla al chino así: “太陽照下來像鉛一般沉重” [Cae el sol tan pesado como el plomo], puesto que el plomo tiene diversas connotaciones y en chino se usa más "el plomo" para indicar su función de escribir: “懷鉛提槧” [lleva el polvo del plomo y las tabletas de madera para escribir en cualquier momento] o de hacer productos cosméticos: “洗盡鉛華 " [dejar de usar los cosméticos y volver a una vida sencilla], pero no se usa para metáforas de peso. Hay otro ejemplo con "madera" y ocurre lo mismo que en la expresión anterior, ya que se requiere más información explicativa. "Yo duermo como un tronco" (Lorca 1954: 886) en español se refiere a que uno está profundamente dormido. En chino se aplica la imagen de la madera para describir a una persona parada “呆若木雞” [ tan tonto como una gallina de madera] o para indicar que 
una persona se siente desesperada “槁木死灰” [árboles secos y cenizas frías], por eso, dormirse como un tronco está fuera de la imaginación de los lectores chinos y sería aconsejable ofrecer más información en el texto meta sobre las connotaciones de la expresión en castellano.

\section{- Desviaciones por falta de conocimiento cultural}

En este apartado comentamos los errores de traducción según la clasificación de Nord (1996). En la traducción china de La casa de Bernarda Alba hemos detectado dos tipos de errores: unos pertenecen al aspecto cultural y los otros al lingüístico. Se trata de errores que no afectan a la comprensión de la historia del texto en general pero pueden confundir a los lectores en partes puntuales. Los errores culturales se refieren a traducciones que no corresponden con los fenómenos culturales españoles y los lingüísticos son traducciones correctas en el sentido de la gramática china pero quieren decir otras cosas que el texto original debido a una comprensión semántica incompleta del texto original. Este último tipo de error se diferencia de la clasificación de Nord (1996) en que el texto meta no presenta defectos lingüísticos sino que comete errores por la mala comprensión del texto original.

Se han localizado errores culturales en el vocabulario de la alimentación y gastronomía, que con frecuencia forma parte del problema de la traducción, puesto que no hay dos culturas que compartan totalmente la misma gastronomía. Sabemos que en España hay bastantes productos cárnicos: jamón, salchichas, chorizos, salchichones, etc. Así, es normal comer estos fiambres junto con el alimento básico, el pan. Sin embargo, en Taiwán el arroz es el alimento básico, la bollería es de tipo artesano, con formas y sabores diversos, incluso con salchichas o perritos calientes. No obstante, los taiwaneses no tienen la costumbre de tomar fiambre cortado con el pan. Por tanto, cuando en la obra de Lorca, aparece "Sale comiendo chorizo y pan." (Lorca 1954: 837), los lectores taiwaneses no tendrán la imagen del chorizo y el pan por separado sino un bollo hecho con chorizo por encima. Suponemos que la versión en inglés está influida por su propia cultura, y no es de extrañar que haya traducido "She enters, eating bread and sausage." (Lorca 1941 y 1955: 157), mientras la versión en chino por un lado, sigue la versión inglesa, y por el otro la costumbre china, pero ha usado una traducción igual que la inglesa: “走進來, 邊吃著臘腸麵包” (Lorca 1970: 125) [Sale comiendo un bollo de salchicha] Es posible que al traductor chino le falten conocimientos sobre cultura occidental y aunque la versión en inglés queda clara, la traducción en chino lo ha traducido a la costumbre taiwanesa.

Otro alimento que crea cierta confusión en la traducción en chino son los garbanzos, que se han convertido en guisantes (楁豆 ) (Lorca 1970: 126). Debería ser "chickpea" pero como el garbanzo prácticamente no se usa en la cocina de EEUU/ Reino Unido, el traductor ha realizado una generalización/adaptación. Creemos que este error deriva de la traducción en inglés y del poco conocimiento de la cultura española de Perng. Por tanto, al traducir de la versión inglesa "peas" (Lorca 1941/55: 157), Perng no se ha dado cuenta de que es raro tomar un puñado de guisantes en la mano, y que en realidad se refería a los garbanzos secos (Lorca 1954: 
838). Este ejemplo ilustra muy bien los peligros de las traducciones indirectas, que pueden llegar a desviarse mucho del TO en la medida en que no pueden diferenciar lo que constituye parte del TO de lo que es una reinterpretación del primer traductor.

\section{- Fallos debidos a aspectos lingüísticos y a la traducción intermedia}

Las connotaciones también son elementos que afectarán a la representación de la idea original del dramaturgo en la traducción de objetos concretos y abstractos como las creencias.

Por otro lado, una traducción indirecta siempre vendrá influida por una tercera lengua y cultura; ya no solo se trata del texto original y del texto meta. De este modo, es posible que la traducción final, al intentar expresar las posibles connotaciones de la traducción intermedia no acabe por acertar con la idea original totalmente.

Hemos hallado ejemplos de este tipo y los hemos considerado errores de lengua, porque a consecuencia de la desviación lingüística se confunde el texto original.

Durante el luto del marido de Bernarda, la familia encierra a la abuela que gritar e intenta salir fuera. La abuela dice entonces a la criada que Bernarda le ha dado el agua de lavar los platos y la comida para los perros. En el texto original Martirio ha comentado a la criada que su abuela Josefa "¡Tiene mala intención!” (Lorca 1954: 136). La traducción en inglés pone "She's mean." (Lorca 1941 y 1955: 165) como una crítica al carácter de la abuela, en vez de un comentario de que lo que ha hecho es por la mala intención que tiene. Mientras en chino la traducción resulta “她好不要臉” [iQué descarada!] (Lorca 1970: 136) Entendemos que tanto la traducción en inglés como la versión en chino son comentarios negativos, sin embargo, ninguna de las dos se corresponde con la idea del texto original. En nuestra opinión, una traducción equivalente al texto original en chino debería ser “她不懷好意” [Tiene mala intención]. En una frase tan corta la traducción directa del castellano funcionaria sin problemas.

Otro ejemplo similar es la expresión "con intención" (Lorca 1954: 851 y 889) que se han convertido en “意味深長” [significante] (Lorca 1970: 137 y 168) en la versión china, una traducción que no tiene que ver con el texto original. Mientras comparamos las traducciones en inglés, "meaningfully" (Lorca 1941 y 1955: 165) y "with meaning" (Lorca 1941 y 1955: 188) entendemos que esa equivocación en chino también procede indudablemente del texto intermedio inglés.

Otro ejemplo es una interferencia lingüística que procede de la versión en inglés, no porque la versión inglesa sea problemática, sino porque la traducción en chino se ve afectada por la gramática inglesa. Después del funeral del marido de Bernarda, la criada preguntó si había suficientes sillas en casa para los parientes y Poncia contestó "Sobran" (Lorca 1954: 839). En la traducción inglesa dice: "More than enough" (Lorca 1941 y 1955: 165) y la traducción en chino sigue el mismo formato lingüístico: “太夠了” [más que suficiente] (Lorca 1970: 127). Sin embargo, en chino no se dice así sino “還有剩呢!” [sobran] o “夠” [bastante] como una respuesta para expresar que hay suficientes sillas en casa. 
Finalmente presentamos ejemplos donde hay problemas en la lengua de llegada. La tarea de traducción requiere una competencia lingüística tanto de la lengua del texto original como de la traducida. No cabe duda de la alta capacidad en chino de Perng como hablante nativo, aunque también es cierto que se encuentran unas traducciones poco fluidas.

Así pues, encontramos problemas de tono: "Le he abierto la orza de los chorizos", (Lorca 1954: 838) dice Poncia para indicar un hecho. En la traducción del chino debería decir “我已經把臘腸罐打開了” [Le he abierto la orza de los chorizos] con tono equivalente, puesto que “我打開了臘腸罐” [Abro la orza de los chorizos] (Lorca 1970: 126) sonaría como si estuviera contando la historia a otra persona y se esperase más información: "Abro la orza del los chorizos y..."

Otro ejemplo de la traducción inusual, que podría ser un invento del traductor y resultado de la influencia del dialecto de Taiwán, es la traducción de Perng de "cállate" (Lorca 1954: 844) como “放安靜” (Lorca 1970: 131). Aunque en el dialecto taiwanés se utiliza el mismo verbo “放” para expresar que se requiere un respeto: “放㑝重”, no es común en este uso para pedir silencio.

Hay también traducciones al chino donde se aplica la traducción literal sin considerar que hay otro significado derivado de una misma palabra. Para indicar un carácter poco amable, en español y en inglés se usa una misma palabra con diferente categoría gramatical: "Seca" (Lorca 1954: 869) y "dryly" (Lorca 1941/ 55: 177). La traducción en chino no se entiende, puesto que en chino no se aplica "seco/a" para el carácter de personas como en la traducción, “乾澀地” (Lorca 1970: 152) sino que se utiliza una palabra derivada del “frío": “冷漠” [seco/a] para indicar una actitud poco simpática. Algo, que, por otro lado también se hace en castellano e inglés y que, por tanto debería ser de sobras conocido por el traductor.

\section{Conclusiones}

Revisando la época de la publicación de La casa de Bernarda Alba en chino vemos que, en efecto, esta traducción resultó ser muy valiosa como pionera que introdujo las obras de Lorca a los lectores chinos. Ya que en esa época todavía no había personas capacitadas para realizar una traducción directa del español al chino, la traducción de Perng ofreció la oportunidad de ampliar la visión literaria en Taiwán donde todavía hoy reinan las obras de autores anglosajones. Sin embargo, hay cierta dificultad a la hora de fomentar el gusto por la lectura teatral, las traducciones teatrales nunca han obtenido un lugar central dentro de la traducción literaria taiwanesa.

Con todo, a partir de los años noventa, las traducciones directas de las novelas hispanoamericanas han empezado a crecer en número y hoy en día podemos encontrar en las librerías novelas traducidas al chino por diferentes traductores.

A partir de la traducción indirecta de Perng, hemos notado que la discrepancia entre dos culturas lejanas, incluso en la vida cotidiana, resulta problemática para la traducción pero también son las marcas imprescindibles que llevan los colores 
autóctonos de cada cultura y que salpican los intereses propios frente a la globalización reinante.

Cuando estudiamos la traducción de los nombres de personas en la traducción de Perng, descubrimos que las reflexiones en Perng (1997) y (2005) no coinciden con los nombres traducidos en La casa de Bernarda Alba. Hemos deducido que la discrepancia de las técnicas de traducción aplicada sea posiblemente debida a la distancia en el tiempo. Cuando Perng realizó la traducción de La casa de Bernarda $A l b a$ en 1970, todavía era un principiante sin mucha experiencia, por tanto, sus ideas sobre la tarea traductora se ceñían al seguimiento del texto original debido a que la autoridad de un texto original en los años setenta era una creencia indiscutible. Así, hemos visto que la traducción de todos los nombres que aparecen en la obra tienen las mismas sílabas que sus nombres en español aunque son largos e impiden una pronunciación fluida en chino en el escenario. Aún así, la semejanza de los dos nombres 潘霞 (Poncia) y 李芭德 (Librada) con nombres chinos nos ha dado la pista de la preferencia del traductor por lograr una sensación de cercanía entre los lectores chinos.

Desde el principio Perng ha recalcado su fidelidad hacia el texto original. Sin embargo, como la traducción de La casa de Bernarda Alba es una traducción indirecta de la versión inglesa, la idea del traductor no garantiza la fidelidad hacia el texto original en español sino una lealtad parcial hacia la traducción en inglés. Los casos de desviación de tipo lingüístico y cultural en que sus traducciones chinas no se corresponden con texto en castellano indican que Perng ni se ha dado cuenta de este fenómeno. Tanto en Perng (2005) como en el prólogo de una traducción directa de la obra de Brecht se describen los defectos de una traducción indirecta.

A nuestro parecer, la necesidad de una traducción directa responde tanto al deseo de ofrecer un auténtico texto original como al de una versión diferente según la temporalidad de cada época, que es una de las nociones de traducción de Perng, mencionada en el apartado 4.2. Por otro lado, una obra es un reflejo sociocultural en el tiempo y su título facilita una idea general para la audiencia. El título de La casa de Bernarda Alba insinúa que la casa es como una sociedad pequeña y una jaula que encierra a la familia de Bernarda, mientras que el subtítulo "Drama de mujeres en los pueblos de España" revela el trasfondo de toda la historia. Sin embargo, Perng ha omitido el subtítulo dejando únicamente el título. Esta elisión está en contra de la idea de lealtad que siempre remarcaba Perng. Como la obra ofrece poca información acerca del trasfondo social, al ni siquiera mencionar la palabra "España", resulta difícil que los lectores chinos se sitúen. Por tanto, corroboramos que la lealtad para Perng es una mera ideología, pero en su práctica de la traducción ha gozado de amplia libertad con resoluciones variadas siempre y cuando la técnica aplicada resulte razonable y no perjudique la idea del texto original.

\section{Referencias bibliográfícas}

Aaltonen, S., Time-Sharing on Stage: Drama Translation in Theatre and Society. Clevedon/ Buffalo/ Toronto/ Sydney: Multilingual Matters 2000. 
Anderman, G., «Drama Translation», en: Baker, MonA, (ed.), Routledge Encyclopedia of Translation Studies. London y New York: Routledge 1998 y 2001, 71-74.

Bassnett, S., «Training Spatial Poetry: An Examination of Theatre Texts in Performance», en Holmes, J., LAmbert, J. \& VAN DEN BroecK, R. (ed.), Literature and Translation: New Perspectives in Literary Studies. Leuven: Acco 1978, 161-176.

BRECHT, B., 《布雷希特戲劇選集, 四川好人 [Antología de las obras de Bertolt Brecht, La buena mujer de Sichuan]» [trad. por Ching-hsi Perng] en YAN, Yuan-shu 顏元叔 (ed.), 淡江西洋現代戲劇譯丵 Serie de traducciones de los teatros occidentales modernos del Colegio Tamkang. Taipei: Jingsheng 驚聲 1973.

BRECHT, B., «四川好人, 高加索灰闌記 [La buena mujer de Sichuan y El círculo de tiza caucásico]», trad. por Perng Ching-hsi 彭鏡禧 y Dscheng Fang-hsiung 鄭芳雄. Taipei: Linkingbooks 聯經 2005 .

Cela, C., La familia de Pascual Duarte. Barcelona: Destino 1942.

CELA, C., 杜瓦特家族 La familia de Pascual Duarte [trad. por 張淑英 Chang Shuying]. Taipei: China Times 1992.

EsQuivel, L., Tan veloz como el deseo. Barcelona: Plaza \& Janéz 2001.

ESQUIVEL, L., 愛情翻譯員 Tan veloz como el deseo, [trad. por 范湲 Fan Yuan]. Taipei: Crown, 2006.

HuRTAdo, A., Traducción y traductología. Introducción a la traductología. Madrid: Cátedra 2001.

Kussmaul, P., Training the Translator. Amsterdam/ Philadelphia: John Benjamins 1995.

LEFEVERE, A., "Translating Literature/ Translated literatura - the State of the Art», en: Zuber, Ortrun (ed.), The Languages of Theatre. Problems in the Translation and Transposition of Drama. Oxford, New York, Toronto, Sydney, Paris y Frankfurt: Pergamon 1980, 153-161.

LorcA, F., «The House of Bernarda Alba», en: Three Tragedies. Blood Wedding. Yerma. The House of Bernarda Alba [trad. por James Graham-Luján y Richard L. O’Connell]. New York: New Directions 1941 y 1955.

LorCA, F., «La casa de Bernarda Alba», en: Lorca, Federico García, Obra Completa, Tomo II. Madrid: Aguilar 1954.

LORCA, F., 《白納德之屋 The House of Bernarda Alba» [trad. por, Ching-hsi Perng 彭鏡禧], en YAN, Yuan-shu 顏元叔 (ed.), 淡江西洋現代戲劇譯叢 [Serie de traducciones de los teatros occidentales modernos del Colegio Tamkang]. Taipei: Jingsheng 驚聲 1970.

Moya, V., La traducción de los nombres propios. Madrid: Cátedra 2000.

NorD, C., Text Analysis in Translation. Ámsterdam/ New York: Rodopi 1988/ 2005.

NoRD, C., «El error en la traducción: categorías y evaluación», en Hurtado A. (ed.), La Enseñanza de la Traducción. Castelló de la Plana: Universitat Jaume I, 1996, 91-107.

OoI, V., «Transcending Culture: a Cantonese Translation and Production of O'Neill's Long Day's Journey into Night», en: Zuber, Ortrun (ed.), The Languages of Theatre. Problems in the Translation and Transposition of Drama. Oxford/ New York/ Toronto/ Sydney/ Paris/ Frankfurt: Pergamon 1980, 51-68.

PERng, Ching-hsi 彭鏡禧, 翻譯文學評論集 [Comentarios de traducciones literarias]. Taipei: Bookman 1997.

PERnG, Ching-hsi, 《苦心孤譯《哈姆雷》》 Translating Hamlet, the Heart Way», 中外文學 Chung Wai Literary Quarterly, 33: 11 (2005), 13-32.

PyM, A., «Translation Error Analysis and the Interface with Language Teaching», en Dollerup, C. y Loddegaard, A. (ed.), The Teaching of Translation. Amsterdam/ Philadelphia: John Benjamins 1992, 278-288.

RuIz, C., La sombra del viento. Barcelona: Planeta 2001. 
RuIZ, C., 風之影 La sombra del viento [trad. por 范湲 Fan Yuan], Taipei: Eurasian 圓神 2004.

YAN, Yuan-shu, «Sobre la Serie de traducciones de los teatros occidentales modernos del Colegio Tamkang 關於淡江西洋現代戲劇譯丵 》, en: YAN, Yuan-shu (ed.), Antología del teatro de Brecht: La Buena mujer de Sezuan 布雷希特戲劇選集, 四川好人, Taipei: Jingsheng 驚聲 1973. 